\title{
Association of rheumatic fever with serum albumin concentration and body iron stores in Bangladeshi children: case-control study
}

M Mostafa Zaman, Nobuo Yoshiike, Mian Abdur Rouf, Sirajul Haque, Anisul Haque Chowdhury, Takeo Nakayama, Heizo Tanaka

Malnutrition in early life may cause metabolic ${ }^{1}$ and immune $^{2}$ imbalance and consequently affect tissue reactivity of the child to group A $\beta$ haemolytic streptococcal infection of the throat, leading to rheumatic fever. ${ }^{1}$ In developing countries protein and iron deficiencies during childhood are common and cause growth retardation, ${ }^{3}$ which is also found in rheumatic fever. ${ }^{4}$ Iron deficiency predisposes to repeated infections, ${ }^{3}$ which are necessary for a rheumatic attack. We examined whether rheumatic fever is associated with serum protein concentrations and body iron stores in Bangladeshi children.

\section{Subjects, methods, and results}

Recruitment of subjects and their socioeconomic background are described elsewhere. ${ }^{4}$ Briefly, 218 consecutive subjects aged 5 to 20 years who had a recent infection with group A $\beta$ haemolytic streptococci were selected in the outpatient clinic of the National Centre for Control of Rheumatic Fever and Heart Diseases, Dhaka. Of them, 60 had rheumatic fever (as defined by the updated Jones criteria 1992) and 158 did not. Fasting convalescent blood samples (3-4 weeks after first contact) were obtained in $44(73 \%)$ subjects with rheumatic fever and 139 (88\%) without. Because the occurrence of rheumatic fever is strongly influenced by age and nutritional deprivation differs between sexes in Bangladesh, 44 subjects without rheumatic fever were randomly matched with cases for age (within one year) and sex.
Haemoglobin concentration and packed cell volume were measured in Bangladesh. Serum samples were stored at $-80^{\circ} \mathrm{C}$, transported to Tokyo on dry ice, and again stored at $-80^{\circ} \mathrm{C}$ until used. Standard methods were used to determine nutritional markers. Transferrin saturation was calculated. Odds ratios and their $95 \%$ confidence intervals were obtained by univariate conditional logistic regression analyses (for matched subjects) for nutritional markers, which were entered into the model as continuous variables. Multiple logistic regression analyses were performed to calculate odds ratios adjusted for several socioeconomic factors that differed significantly between the groups. A measure of inflammation ( $\mathrm{C}$ reactive protein concentration) was also considered as a covariate because it influences the nutritional markers of interest.

The mean (SD) age of the cases and controls was 12.6 (3.2) and 12.5 (3.2) years respectively, with 26 boys in each group. None of the cases had rheumatic heart disease or congestive heart failure. Three cases were presumed to have had an attack of rheumatic fever. The cases had lower serum albumin concentrations and all measures of body iron stores. Logistic regression analyses showed lower risk estimates for one unit increase in these variables (table). However, when confounding by socioeconomic factors and inflammation was taken into account, significance persisted for albumin concentration, packed cell volume, iron stores, and transferrin saturation. Analyses that excluded the three cases that were presumed to have had an attack of rheumatic fever gave similar results.

Mean (SD) concentrations of nutritional markers, with risk of rheumatic fever associated with one unit increase in their serum values

\begin{tabular}{|c|c|c|c|c|c|c|}
\hline \multirow[b]{3}{*}{ Variable } & & & \multicolumn{4}{|c|}{ Risk estimates } \\
\hline & \multicolumn{2}{|c|}{ Mean (SD) } & \multicolumn{2}{|l|}{ Unadjusted } & \multicolumn{2}{|l|}{ Adjusted ${ }^{*}$} \\
\hline & Cases & Controls & Odds ratio $(95 \% \mathrm{Cl})$ & $P$ valuet & Odds ratio $(95 \% \mathrm{Cl})$ & $P$ valuet \\
\hline Total protein (g/l) & $84(6)$ & $82(6)$ & 1.06 (0.98 to 1.15$)$ & 0.17 & $1.02(0.92$ to 1.14$)$ & 0.66 \\
\hline Albumin $(g / l)$ & $44(4)$ & $48(3)$ & 0.72 (0.59 to 0.88$)$ & 0.001 & 0.75 (0.60 to 0.95$)$ & 0.02 \\
\hline \multicolumn{7}{|l|}{ Cholesterol (mmol/l): } \\
\hline Total & $3.55(0.62)$ & $3.64(0.66)$ & $0.80(0.41$ to 1.57$)$ & 0.51 & $1.20(0.46$ to 3.16$)$ & 0.71 \\
\hline $\mathrm{HDL}$ & $0.91(0.21)$ & $1.06(0.24)$ & 0.05 (0.01 to 0.49$)$ & 0.01 & 0.38 (0.03 to 5.48$)$ & 0.48 \\
\hline LDL & $2.15(0.61)$ & $2.05(0.56)$ & 1.44 (0.65 to 3.22$)$ & 0.37 & 1.56 (0.51 to 4.74$)$ & 0.44 \\
\hline Triglycerides (mmol/l) & $1.06(0.46)$ & $1.15(0.42)$ & 0.50 (0.15 to 1.73$)$ & 0.27 & 0.86 (0.15 to 4.82$)$ & 0.87 \\
\hline Haemoglobin $(\mathrm{g} / \mathrm{l})$ & $115(14)$ & $123(12)$ & 0.94 (0.90 to 0.99$)$ & 0.01 & 0.96 (0.91 to 1.02$)$ & 0.16 \\
\hline Packed cell volume (\%) & $37.0(3.9)$ & $39.3(2.4)$ & 0.73 (0.59 to 0.91$)$ & 0.004 & 0.69 (0.50 to 0.97$)$ & 0.03 \\
\hline Iron $(\mu \mathrm{mol} / \mathrm{l})$ & $9(5)$ & $14(6)$ & 0.81 (0.71 to 0.93$)$ & 0.002 & 0.82 (0.68 to 0.97$)$ & 0.02 \\
\hline Total iron binding capacity $(\mu \mathrm{mol} / \mathrm{l})$ & $57(10)$ & $61(8)$ & 0.95 (0.91 to 1.00$)$ & 0.06 & 0.96 (0.91 to 1.04$)$ & 0.43 \\
\hline Transferrin saturation (\%)‡ & $15.8(7.9)$ & $22.8(9.7)$ & 0.89 (0.83 to 0.96$)$ & 0.002 & 0.90 (0.82 to 0.98$)$ & 0.02 \\
\hline
\end{tabular}

HDL=high density lipoprotein; LDL=low density lipoprotein.

*Adjusted for location of residence (urban and rural), parental schooling, number of siblings, and C reactive protein concentration (square root transformed), which were significantly different between cases and controls. However, house type (two categories) was not considered because of its moderate correlation with location of residence $(r=0.51, \mathrm{P}<0.001)$. Other variables that were not considered in the model because of they did not differ significantly were attendance at school (yes, no), rheumatic fever in first degree relatives (yes, no), income (transformed), number of family members, number of people sharing bedroom with the subject, number of people per dwelling room, and results of liver function test (alanine aminotransferase).

†Two tailed.

$\ddagger$ Serum iron concentration/total iron binding capacity $\times 100$.

Department of Epidemiology, Medical Research Institute, Tokyo Medical and Dental University, 2-3-10 Kandasurugadai, Tokyo 101, Japan

M Mostafa Zaman, doctoral student

Takeo Nakayama, assistant professor Heizo Tanaka, professor

Division of Adult Health Science,

National Institute of Health and Nutrition, 1-23-1 Toyama, Tokyo 162

Nobuo Yoshiike, senior researcher

National Centre for Control of

Rheumatic Fever and Heart Diseases, Sher-e-Bangla Nagar, Dhaka 1207, Bangladesh

Mian Abdur Rouf, microbiologist Sirajul Haque, professor of cardiology Anisul Haque Chowdhury, research assistant

Correspondence to: Dr M M Zaman,

National Centre for Control of

Rheumatic Fever

and Heart Diseases,

Sher-e-Bangla

Nagar, Dhaka 1207, Bangladesh

mzaman@bangla.net

BMJ 1998;317:1287-8 


\section{Comment}

All subjects had recent infections with group A $\beta$ haemolytic streptococci. The findings attributable to this particular inclusion criterion may widen our understanding of why most children with such infection escape a rheumatic attack while only a few do not. Our analyses adjusted for socioeconomic factors imply that low serum albumin concentrations and body iron stores may contribute to rheumatic fever. To rule out the possibility that differences in nutritional markers reflect differences in severity of inflammation, we adjusted for $\mathrm{C}$ reactive protein concentration in our analyses.

Iron deficiency might have favoured rheumatic fever by predisposing the cases to frequent infections with group A $\beta$ haemolytic streptococci. The target organ lesions in patients with rheumatic fever predominantly contain $\mathrm{T}$ cells. We speculate that the tissue damage caused by $\mathrm{T}$ cell infiltration may be favoured by protein deprivation because $\mathrm{T}$ cell mediated immunological functions become exaggerated under mild to moderate chronic protein or protein energy deprivation. ${ }^{5}$ Although case-control studies have inherent limitations, our results suggest that deficiency of albumin and iron is linked to susceptibility to rheumatic fever.

The preliminary results of this work were presented at the 14th congress of rheumatology of the International League of Asso- ciations of Rheumatologists in Singapore, 8-13 June 1997. Fouzia Hasin, Amiruzzaman Khan, Billah Khan, and Mohammad Osman helped in recruiting the subjects. MMZ is a Monbusho (Japanese ministry of education, science, sports, and culture) scholar.

Contributors: MMZ initiated and coordinated the study, formulated study hypothesis, designed the protocol, discussed core ideas, analysed data, interpreted results, and wrote the manuscript. NY initiated the study, prepared the protocol, interpreted results, and revised the manuscript. MAR revised the laboratory aspect of the protocol, collected samples, and monitored the quality of diagnostic laboratory tests. SH revised the clinical aspect of the protocol, coordinated subject recruitment, and validated clinical diagnosis. AHC executed subject recruitment, data documentation, and quality control. TN helped in data analyses and revised critically the intellectual content of the manuscript. HT critically reviewed the protocol and manuscript and approved the final version for publication.

Funding: This work was partially supported by a grant (4C-2) for international health cooperation research from the Ministry of Health and Welfare, Japan.

Conflict of interest: None.

Coburn AF. The continuous association of poverty with intensity of rheumatic manifestations. Am J Med Sci 1960;240:687-99.

2 Aryanpur-Kashani I. On rheumtic fever in children. Am Heart $J$ 1980;100:942-3.

3 Gibson RS. Principle of nutritional assessment. New York: Oxford University Presss, 1990.

4 Zaman MM, Yoshiike N, Chowdhury AH, Nakayama T, Yokoyama T, Faruque GM, et al. Nutritional factors associated with rheumatic fever: the Nutriheart study. J Trop Pediatrics 1998;44:142-7.

5 Cooper WC, Good RA, Mariani T. Effects of protein insufficiency on immune responsiveness. Am J Clin Nutr 1974;27:647-64.

(Accepted 16 April 1998)

\section{Biliary heavy metal concentrations in carcinoma of the gall bladder: case-control study \\ V K Shukla, A Prakash, B D Tripathi, D C S Reddy, S Singh}

Departments of Surgery and Community

Medicine, Institute of Medical Sciences, Banaras Hindu University, Varanasi - 221005 , India

V K Shukla,

professor of surgery

A Prakash,

senior resident in

surgery

D C S Reddy,

reader in community

medicine

S Singh,

research associate in

surgery

Department of

Botany, Faculty of

Science, Banaras

Hindu University

B D Tripathi,

reader

Correspondence to: Professor Shukla

vkshukla@banaras.

ernet.in

BMJ 1998;317:1288-9
Carcinoma of the gall bladder is the third most common malignancy of the gastrointestinal tract in the eastern Uttar Pradesh and western Bihar regions of India. ${ }^{1}$ The two regions lie down stream of the river Ganges, which is the main source of drinking, bathing, and irrigation water in this part of India and receives untreated domestic sewage and industrial and agricultural effluent. High concentrations of cadmium have been reported in sewage, irrigation water, and vegetables grown in the area, and higher concentrations of heavy metals than recommended by the World Health Organisation have been reported in water from this region. Heavy metals as environmental pollutants have been implicated in human carcinogenesis. ${ }^{2}$ These metals, especially cadmium, are excreted and concentrated in the hepatobiliary system. ${ }^{3}$

We investigated whether gallbladder cancer was associated with exposure to heavy metals and hence high biliary concentrations.

\section{Patients, methods, and results}

The study was carried out in 96 patients with gallbladder diseases admitted to the surgical unit of the University Hospital, Varanasi, from January 1995 to
March 1996. All these patients were from the same geographical area. Thirty eight patients had histologically diagnosed carcinoma of the gall bladder (mean age 53.5 years; 11 men and 27 women; 25 had associated calculi) and 58 had gall stones (control group; mean age 48.3 years; 14 men and 44 women). Bile (10 $\mathrm{ml}$ ) was taken by needle aspiration from the gall bladder of all patients at the time of surgery for estimation of cadmium, chromium, and lead concentrations. The sample was stored at $-20^{\circ} \mathrm{C}$ until analysed by the method described in the 1982 manual for PerkinElmer's model 2380 atomic absorption spectrophotometer. Student's $t$ test was carried out using MSTAT software.

The figure shows that mean biliary concentrations of cadmium, chromium, and lead were significantly higher in patients with carcinoma of the gall bladder than in those with gall stones (cadmium: 0.19 (SE 0.07) $\mathrm{mg} / \mathrm{l}$ ข 0.09 (0.04) $\mathrm{mg} / \mathrm{l}$, difference 0.10 (95\% confidence interval 0.08 to 0.12$), t=11.63$, $\mathrm{df}=93.63$, $\mathrm{P}<0.001$; chromium: 1.26 (0.06) $\mathrm{mg} / \mathrm{l} v 0.55$ (0.03) $\mathrm{mg} / \mathrm{l}$, difference 0.71 (0.58 to 0.84$), t=9.84, \mathrm{df}=57.45$, $\mathrm{P}<0.001$; lead: 58.38 (1.76) mg/l v 3.99 (0.43) mg/l, difference 54.4 (50.7 to 58.0 ), $t=30.07, \mathrm{df}=41.43$, $\mathrm{P}<0.001)$ 